\title{
Kooperation zahlt sich aus
}

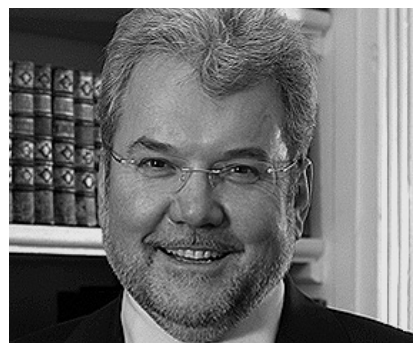

VON ECKHARD EYER

Eckhard Eyer berät Einrichtungen der Sozialwirtschaft bei der Gestaltung und Einführung von Grund- und Leistungsentgeltsystemen und arbeitet dabei sowohl mit Geschäftsleitungen wie mit Betriebsräten zusammen. In betrieblichen Einigungsprozessen wird er als Mediator hinzugezogen. Er ist Gründer des FAIRInstituts für praktische WirtschaftsMediation, Autor zahlreicher Fachpublikationen und Lehrbeauftragter an der Universität Trier.

Internet http://www.eyer.de

\author{
Bei den immer neuen Herausforderungen, die auf die \\ Sozialwirtschaft zukommen, und den damit verbunde- \\ nen Innovationen ist die konstruktive Zusammenarbeit \\ mit dem Betriebsrat entscheidend für die Geschwindig- \\ keit und den Verlauf von Veränderungsprozessen und \\ damit für den Erfolg des Unternehmens.
}

Damit Veränderungsprozesse auf einem hohen fachlichen Niveau zügig verlaufen können, müssen die Mitarbeiterinnen und Mitarbeiter beteiligt werden. Das gilt ebenso für die Vertreter der Mitarbeitenden, die Betriebs- und Personalräte oder die Mitarbeitervertreter (in diesem Beitrag alle »Betriebsrat « genannt)

Arbeitgeber und Betriebsrat vertreten teilweise unterschiedliche Interessen, das zeigt sich am deutlichsten bei den Gehaltsregelungen. Was für den Arbeitgeber Kosten sind, ist für die Arbeitnehmer Einkommen. Das Betriebsverfassungsgesetz (BetrVG) regelt die Zusammenarbeit zwischen Arbeitgeber und Betriebsrat. Trotz der unterschiedlichen Interessen fordert es in $\mathbb{S}$, Absatz 1 eine vertrauensvolle Zusammenarbeit mit dem Ziel des Wohls der Arbeitnehmer und des Betriebes.

Das Betriebsverfassungsgesetz regelt differenziert die Rechte und Pflichten der Mitarbeitenden und ihrer gewählten Vertreter. Der Betriebsrat hat gegenüber dem Arbeitgeber Unterrichtungs-, Beratungsrechte und Mitbestimmungsrechte. Letztere werden insbesondere in \87 Absatz 1 BetrVG geregelt.

Wie Arbeitgeber und Betriebsrat ihre Zusammenarbeit gestalten, ist in den Betrieben sehr unterschiedlich und von einer Reihe von Faktoren abhängig, nicht zuletzt vom Selbstverständnis der Betriebsparteien, ihrer Informationspolitik, der Form ihrer Zusammenarbeit und dem Umgang miteinander.
Die betriebliche Praxis zeigt: Das Selbstverständnis der Betriebsparteien wird stark davon prägt, wie man auf sein Gegenüber zugeht und mit ihm zusammenarbeitet. Es ist wichtig, sich als Arbeitgeber und Betriebsrat zu prüfen, welches Verständnis man vom Gegenüber hat und wie man die Zusammenarbeit gestalten will.

Die Sicht der Arbeitgeber auf ihre Betriebsräte ist differenziert, sie reicht von

- einer sehr kritischen Sicht auf die Betriebsräte, weil die Existenz eines Betriebsrates im eigenen Unternehmen eine Misstrauensäußerung gegenüber dem Arbeitgeber ist, über

- eine Sicht auf die Betriebsräte nach dem Motto » Betriebsratsarbeit ist nur unproduktive Doppelarbeit « und

- der Betriebsrat ist eine Notwendigkeit, die der demokratischen Grundordnung geschuldet ist,

- bis hin zur Wahrnehmung des Betriebsrates als Ko-Manager, mit dem man gemeinsam Veränderungsprozesse aufgrund des Sachverstands schneller und konstruktiver sowie kollektivrechtlich für alle Mitarbeiterinnen und Mitarbeiter verbindlich lösen kann.

Betriebsräte - als Einzelne und als Gremium - haben aufgrund ihrer persönlichen und betrieblichen Erfahrun- 
gen als Arbeitnehmer und als Betriebsrat ein Verständnis von ihrer Betriebsratsarbeit und von dem entwickelt, was sie damit erreichen wollen. Sie reicht

- von der Wahrnehmung des Arbeitgebers als einem vorwiegend an Rendite orientierten Menschen, der die Mitarbeitenden überfordert und manchmal übervorteilt, so dass er sich als Betriebsrat schützend vor die Kollegenschaft stellen muss,

- über einen paternalistischen Arbeitgeber, der durch seine nette und fürsorgliche Art den Betriebsrat als engagierten Vertreter der Mitarbeiterinteressen überflüssig macht,

- bis hin zu einem Arbeitgeber, der den Kunden, den Eigentümern und den Mitarbeitern gerecht werden will, das aber nur kann, wenn der Betriebsrat als Gegenüber die Interessen der Mitarbeiter angemessen vertritt und faire und zukunftsfähige Lösungen mit erarbeitet.
Der Auftrag des Betriebsverfassungsgesetzes wird gerne so missverstanden, dass die Betriebsräte nur für das Wohl der Arbeitnehmer zuständig sind und der Arbeitgeber nur für das wirtschaftliche Wohl des Betriebes zuständig ist. Der gemeinsame Weg der vertrauensvollen Zusammenarbeit zum Wohle der Arbeitnehmer und des Betriebes ist zunächst schwieriger, auch weil ungewohnt. Er ist aber auch vielversprechend.

\section{Informationen stärken Vertrauen}

Die Informationspolitik des Arbeitgebers gegenüber den Mitarbeitern und dem Betriebsrat ist ein wichtiger Hebel zur Gestaltung der Zusammenarbeit in der Hand des Arbeitgebers.

Informiert er beispielsweise Betriebsrat und Mitarbeitende immer gleichzeitig und fordert dann den Betriebsrat zu einer Stellungnahme auf, kann der nur schwer angemessen rea- gieren. Er wird sicherheitshalber grundsätzlich Vorbehalte haben, Kritik äußern und sich auf »Das ist zu prüfen « zurückziehen. Wenn man sich als Interessenvertreter einmal inhaltlich tendenziell festgelegt hat, wird eine Änderung der Meinung schnell zur Frage des Gesichtsverlustes. Sollte der Betriebsrat von den Mitarbeitenden als schwach wahrgenommen werden, wird er versuchen, sich an anderer Stelle als starker Interessenvertreter der Kollegenschaft zu profilieren.

Werden der Betriebsrat oder seine beiden Vorsitzenden dagegen über eine neue Situation und den daraus resultierenden Handlungsbedarf rechtzeitig informiert, dann kann der Betriebsrat sich beraten, sachlich fundiert in einer Mitarbeiterinformationsrunde oder Mitarbeiterversammlung auftreten und seine Rolle konstruktiv wahrnehmen.

Doch sollte der Arbeitgeber Betriebsrat und Mitarbeiterschaft nicht nur in schwierigen Zeiten angemessen

\section{Das sagt das Betriebsverfassungsgesetz}

\section{§2 Stellung der Gewerkschaften und Vereinigungen der Arbeit- geber}

(1) Arbeitgeber und Betriebsrat arbeiten unter Beachtung der geltenden Tarifverträge vertrauensvoll und im Zusammenwirken mit den im Betrieb vertretenen Gewerkschaften und Arbeitgebervereinigungen zum Wohl der Arbeitnehmer und des Betriebs zusammen.

(...)

\section{\$87 Mitbestimmungsrechte}

(1) Der Betriebsrat hat, soweit eine gesetzliche oder tarifliche Regelung nicht besteht, in folgenden Angelegenheiten mitzubestimmen:

1. Fragen der Ordnung des Betriebs und des Verhaltens der Arbeitnehmer im Betrieb;

2. Beginn und Ende der täglichen Arbeitszeit einschließlich der Pausen sowie Verteilung der Arbeitszeit auf die einzelnen Wochentage;

3. vorübergehende Verkürzung oder Verlängerung der betriebsüblichen Arbeitszeit;

4. Zeit, Ort und Art der Auszahlung der Arbeitsentgelte;

5. Aufstellung allgemeiner Urlaubsgrundsätze und des Urlaubsplans sowie die Festsetzung der zeitlichen Lage des Urlaubs für einzelne Arbeitnehmer, wenn zwischen dem Arbeitgeber und den beteiligten Arbeitnehmern kein Einverständnis erzielt wird;

6. Einführung und Anwendung von technischen Einrichtungen, die dazu bestimmt sind, das Verhalten oder die Leistung der Arbeitnehmer zu überwachen;
7. Regelungen über die Verhütung von Arbeitsunfällen und Berufskrankheiten sowie über den Gesundheitsschutz im Rahmen der gesetzlichen Vorschriften oder der Unfallverhütungsvorschriften;

8. Form, Ausgestaltung und Verwaltung von Sozialeinrichtungen, deren Wirkungsbereich auf den Betrieb, das Unternehmen oder den Konzern beschränkt ist;

9. Zuweisung und Kündigung von Wohnräumen, die den Arbeitnehmern mit Rücksicht auf das Bestehen eines Arbeitsverhältnisses vermietet werden, sowie die allgemeine Festlegung der Nutzungsbedingungen;

10. Fragen der betrieblichen Lohngestaltung, insbesondere die Aufstellung von Entlohnungsgrundsätzen und die Einführung und Anwendung von neuen Entlohnungsmethoden sowie deren Änderung;

11. Festsetzung der Akkord- und Prämiensätze und vergleichbarer leistungsbezogener Entgelte, einschließlich der Geldfaktoren;

12. Grundsätze über das betriebliche Vorschlagswesen;

13. Grundsätze über die Durchführung von Gruppenarbeit; Gruppenarbeit im Sinne dieser Vorschrift liegt vor, wenn im Rahmen des betrieblichen Arbeitsablaufs eine Gruppe von Arbeitnehmern eine ihr übertragene Gesamtaufgabe im Wesentlichen eigenverantwortlich erledigt.

(2) Kommt eine Einigung über eine Angelegenheit nach Absatz 1 nicht zustande, so entscheidet die Einigungsstelle. Der Spruch der Einigungsstelle ersetzt die Einigung zwischen Arbeitgeber und Betriebsrat. 
informieren, sondern ständig für einen regelmäßigen Informationsfluss sorgen. Dadurch können auch die Beziehungen gepflegt und kleine Probleme rechtzeitig besprochen werden - bevor sie zu großen Problemen werden.

Durch eine kluge Gestaltung der zielgruppengerechten und zeitlich sinnvoll terminierten Information lässt sich eine Reihe von Irritationen in der $\mathrm{Zu}$ sammenarbeit vermeiden. Wenn die Betriebsräte dann auch die Zeit nutzen, um sich zu informieren und bei Bedarf zu qualifizieren, stärkt dies das Vertrauen der Betriebsparteien zueinander.

\section{Lösungen systematisch erarbeiten}

Die Herausforderungen der Sozialwirtschaft treffen nicht nur einzelne Einrichtungen, sondern die ganze Branche. Von daher ist es häufig naheliegend, eine Lösung aus einer befreundeten Einrichtung zu kopieren oder die vom Arbeitgeberverband empfohlene Regelung $\mathrm{zu}$ übernehmen. Spannend wird es, wenn der Betriebsrat lieber die von sei- ner Gewerkschaft empfohlene Regelung umsetzen will. Aus der Frage: »Was dient dem Wohl der Einrichtung und der Mitarbeitenden? « wird nicht selten die Frage: »Wer setzt sich durch? «

In der betrieblichen Praxis hat es sich bewährt, systematisch vorzugehen und Fragestellungen in drei Phasen abzuarbeiten. Dies gilt besonders in schwierigen Situationen und bei komplexen Herausforderungen. Die drei Phasen sind die

\section{- qualitative Phase \\ - quantitative Phase und \\ - rechtliche Phase.}

Nach der Analyse der Situation erarbeiten sich Arbeitgeber und Betriebsrat in der ersten, der qualitativen Phase eine Vorstellung über die Ziele, die sie mit der Lösung erreichen wollen. Sie analysieren, welche Lösungsmöglichkeiten sie haben, und nehmen eine erste Bewertung der Lösungsalternativen vor.

In der zweiten, der quantitativen Phase werden die möglichen Lösungen auf ihre Realisierbarkeit hinsichtlich
Kosten und Nutzen sowie Auswirkungen auf Mitarbeiter und Einrichtung berechnet und die praktische Umsetzbarkeit geprüft.

Erst wenn beide Betriebsparteien wissen, was sie gemeinsam wollen und welche konkreten Auswirkungen es hat, wird das Ergebnis in der dritten, der rechtlichen Phase in einer Betriebsvereinbarung festgeschrieben.

Dieses systematische Vorgehen sichert die Transparenz des Prozesses für alle Beteiligten, reduziert unfruchtbare Diskussionen und führt dazu, dass sich die Teilnehmer beider Betriebsparteien im Prozess fachlich qualifizieren. Sie können - das zeigt die Erfahrung - nach Abschluss der Betriebsvereinbarung aufgrund der geführten Diskussionen die Ergebnisse ihrer gemeinsamen Arbeit gut an die Mitarbeiterinnen und Mitarbeiter transportieren und vor ihnen vertreten.

Der Erfolg, den Arbeitgeber und Betriebsrat gemeinsam haben, führt auch schrittweise zu einer neuen Form der konstruktiven Zusammenarbeit. Die Erwartungen der Mitarbeiter an Be-

\section{Konstruktiver Gestaltungsprozess}

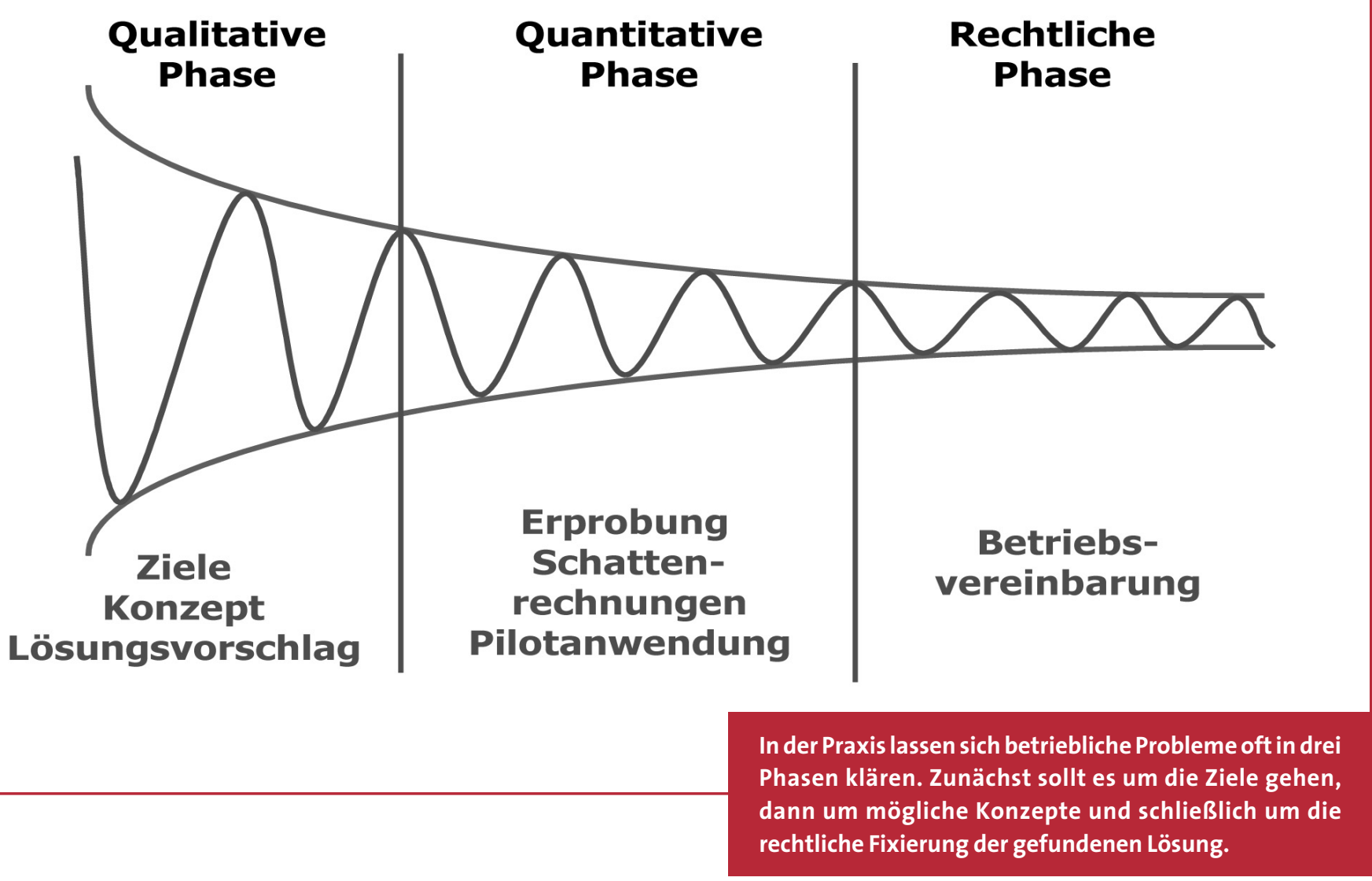


triebsrat und Arbeitgeber sowie der Umgang, den sie miteinander pflegen, ändern sich auch. Das Profilieren auf Kosten des jeweils anderen weicht einem konstruktiven Miteinander, das vom gemeinsamen Erfolg lebt.

In einer Reihe von Betrieben der Sozialwirtschaft konnten nach dieser Methode eine Reihe von Aufgaben erfolgreich bearbeitet werden, beispielsweise:

- Übergang von der 5-Tage-Woche auf die 6-Tage-Woche

- Änderung des Entgeltsystems, weg vom BAT oder TVöD hin zu einem betrieblichen Entgeltsystem

- Einführung eines Leistungsentgeltes

- Abschluss einer Betriebsvereinbarung über die Kürzung der Jahressonderzahlungen im Rahmen tariflicher Härteklauseln

- Einführung von Jahresarbeitszeitkonten mit Lebensarbeitszeitkonten

\section{Von anderen lernen - aber richtig}

Wie bereits erwähnt, treffen die aktuellen Herausforderungen nahezu alle Einrichtungen der Branche. Es kann deshalb sinnvoll sein, von den Erfahrungen anderer Träger zu profitieren. Das sollte nicht einfach dadurch geschehen, dass man sich deren Betriebsvereinbarungen anschaut und sie dem Gegenüber zur Übernahme vorlegt. Sondern dadurch, dass Arbeitgeber und Betriebsrat gemeinsam eine Einrichtung, die die gleiche Herausforderung erfolgreich gemeistert hat, besuchen und sich dort von Arbeitgeber und Betriebsrat gemeinsam erklären lassen

- wie die Ausgangssituation war

- wie man vorging

- welche Probleme auftraten

- wie man sie gelöst hat und

- zu welchem Ergebnis man kam.
Anschließend sollten sowohl die Arbeitgeber als auch Betriebsräte ausreichend Zeit haben, um das gemeinsam Vorgestellte in getrennten Gesprächen zu erörtern. Dieses Vorgehen gibt beiden Parteien die gleichen Hintergrundinformationen und die handelnden Personen qualifizieren sich in den Gesprächen. Durch das gemeinsame Handeln entsteht Vertrauen. Arbeitgeber und Betriebsrat gewinnen Argumente und eine Referenz, warum das, was sie dann tun, richtig ist. Dieses Vorgehen hat sich vielfach in Einrichtungen bewährt und wurde insbesondere dort angewandt, wo die Zusammenarbeit angespannt war.

\section{Blockaden lösen}

Mitunter ist die Zusammenarbeit der Betriebsparteien so destruktiv, dass sinnvolle Lösungen bei den anstehenden Herausforderungen nicht mehr al-

\section{Basisinformation Betriebsrat}

Betriebsräte werden in Betrieben mit in der Regel mindestens fünf Arbeitnehmern gewählt. Der Betriebsrat ist die unabhängige, betriebliche Interessenvertretung aller Beschäftigten, ausgenommen leitende Angestellte. Sind mindestens fünf Jugendliche unter 18 oder Auszubildende unter 25 Jahren beschäftigt, wird zusätzlich eine Jugend- und Auszubildendenvertretung gewählt. Gesetzliche Grundlage für die Wahl und die Aufgaben des Betriebsrat ist das Betriebsverfassungsgesetz (BetrVG) von 1972, das 2001 novelliert und an veränderte Unternehmensstrukturen, neue Formen der Arbeitsorganisation und der Beschäftigung wie Leiharbeit angepasst wurde. Besondere Rechte gelten nun bei Betriebsspaltungen und Betriebszusammenlegungen, und auch bei der Beschäftigungssicherung und Qualifizierung haben Betriebsrat stärkere Rechte. Das Wahlverfahren für Betriebsräte wurde in Betrieben mit bis zu 50 Beschäftigten vereinfacht. Die Beteiligungsrechte des Betriebsrats sind unterschiedlich stark: Sie reichen von reinen Informationspflichten über Beratungspflichten des Arbeitgebers, bei den Betriebsräte unter bestimmten Voraussetzungen Widerspruch einlegen können, bis zu Mitbestimmungsrechten, bei denen im Fall einer Nichteinigung eine Einigungsstelle entscheidet. Betriebsräte sind verpflichtet, gemeinsam mit dem Arbeitgeber die Gleichbehandlung aller Beschäftigten zu sichern und insbesondere Minderheiten oder Schwächere zu unterstützen und zu schützen. Zu den allgemeinen Aufgaben des Betriebsrats zählen u. a. die Überwachung der zugunsten der Arbeitnehmer geltenden Gesetze, Verordnungen, Unfallverhütungsvorschriften, Tarifverträge und Betriebsvereinbarungen, die Durchsetzung der tatsächlichen Gleichstellung von Frauen und Männern, die Förderung der Vereinbarkeit von Familie und Erwerbstätigkeit, der Beschäftigung älterer Arbeitnehmer, der
Beschäftigungssicherung, der Eingliederung Schwerbehinderter und anderer besonders schutzbedürftiger Personen, der Integration ausländischer Arbeitnehmer, von Maßnahmen des betrieblichen Arbeits- und Umweltschutzes und zur Bekämpfung von Rassismus und Fremdenfeindlichkeit. Mitbestimmungsrechte hat der Betriebsrat bei Einstellungen, Kündigungen, Versetzungen und Um- oder Eingruppierungen. Als "Herzstück der Betriebsverfassung " gilt die Mitbestimmung in sozialen Angelegenheiten, beispielsweise Beginn und Ende der täglichen Arbeitszeit, Pausenregelungen, betriebliche Sozialleistungen etc. Betriebsvereinbarungen zwischen Betriebsrat und Arbeitgeber sind eine wichtige und in der Praxis häufige Form, Mitbestimmungsrechte auszuüben, allerdings dürfen sie Tarifverträge nicht unterlaufen. Für Betriebsrat und Arbeitgeber gilt der Grundsatz der vertrauensvollen Zusammenarbeit, beispielsweise kann der Betriebsrat nicht zum Streik aufrufen. Für Beschäftigte im öffentlichen Dienst gilt statt des BetrVG das Personalvertretungsrecht des Bundes und der Länder (Personalrat). Vom BetrVG ausgenommen sind Religionsgemeinschaften, in denen die Vorschriften über Mitarbeitervertretungen einen gewissen Ausgleich schaffen. Eingeschränkt gilt das BetrVG in Tendenzbetrieben, die erzieherischen, karitativen oder konfessionellen Bestrebungen dienen.

Barbara Kahler

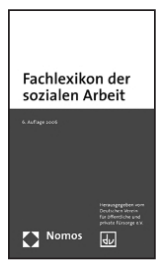

Quelle: Deutscher Verein für öffentliche und private Fürsorge e. V. (Hg.): Fachlexikon der sozialen Arbeit. 6. Auflage. Nomos Verlagsgesellschaft, Baden-Baden 2007. 1.195 Seiten. 44,- Euro. ISBN 9783-8329-1825-5. Seite $130 \mathrm{f}$. 
leine erarbeitet werden können und die Einigungsstelle die Lösung bringen soll.

In der Einigungsstelle wird " an der Sache" gearbeitet, auch wenn die Blockaden auf der Beziehungsebene liegen. Es kann deshalb in Einzelfällen wenn beide Betriebsparteien die Situation gleich einschätzen und eine gemeinsame Lösung wollen - sinnvoll sein, die Sachebene zu verlassen und die Beziehungsebene zu thematisieren.

Diesen Weg wählte man in einem Unternehmen, in dem fünf Betriebsvereinbarungen gekündigt waren und zwei Einigungsstellen arbeiteten. Man unterbrach alle Verhandlungen für die Dauer eines Moratoriums und thematisierte - unter Anleitung eines externen Dritten - die Form der bisherigen $\mathrm{Zu}$ sammenarbeit. Dabei kamen u. a. folgende Punkte zur Sprache:

- Freud und Leid der Zusammenarbeit

- das Selbstverständnis der Parteien

- die Gründe für das Misstrauen gegeneinander

- die Geschichte der Zusammenarbeit und

- die Vorstellungen von einer vertrauensvollen Zusammenarbeit.

Am Ende mehrerer Workshops wurden Spielregeln für die zukünftige $\mathrm{Zu}$ sammenarbeit formuliert und es wurde beschlossen, diese bei einem der sieben anstehenden Themen gleich anzuwenden. Nachdem die erste Betriebsvereinbarung unterzeichnet war, folgten die anderen sechs innerhalb des nächsten halben Jahres. Die Einigungsstellen wurden aufgelöst.

\section{Fazit}

Die konstruktive Zusammenarbeit zwischen Management und Betriebsrat ist von einer Reihe von Faktoren abhängig, die alle veränderbar und von den Betriebsparteien gestaltbar sind.

Mit der Informationspolitik kann der Arbeitgeber entscheidenden Einfluss auf die Zusammenarbeit nehmen. Die Transparenz der Veränderungsund Gestaltungsprozesse und das gemeinsame systematische Vorgehen sind für den Erfolg wichtig. Das einfache Kopieren von Betriebsvereinbarungen anderer Einrichtungen kann zu Blockaden führen, deshalb ist die Übernahme von Lösungen Dritter nur dann zu emp-

fehlen, wenn diese von beiden Betriebspartnern verstanden werden.

Falls längerfristig Blockaden vorliegen, können diese - unabhängig von der fachlichen Thematik - besprochen und ihre Ursachen ergründet werden. Hierzu gehören eine hohe Bereitschaft zur Offenheit und das vorher erklärte Ziel beider Betriebsparteien, die $\mathrm{Zu}$ sammenarbeit zu verbessern.

\section{-}

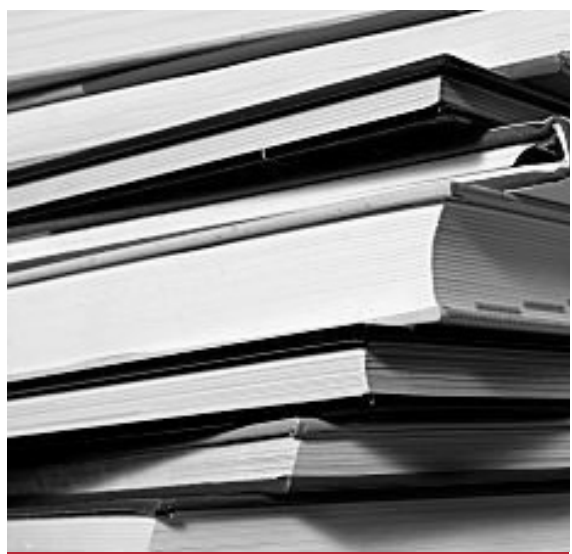

\section{Literatur}

Eyer, E.: Report Wirtschaftsmediation, Krisen meistern durch professionelles Konfliktmanagement, 2. erweiterte Auflage, Symposion Verlag, Düsseldorf 2003.

Eyer, E.: Innovative Entgeltsysteme, Mitarbeiter nach Tätigkeit, Leistung und Erfolg vergüten, Vincentz Verlag, Hannover 2010.

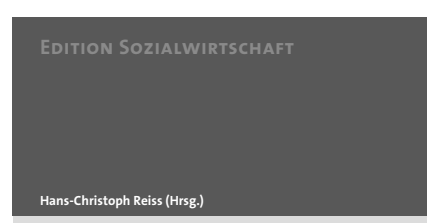

Steuerung von Sozial- und Gesundheitsunternehmen

X Nomos

\section{Steuerung von Sozial- und Gesundheitsunter- nehmen}

Herausgegeben von Prof. Dr. Hans-Christoph Reiss

2010, 404 S., brosch., 69,-€ ISBN 978-3-8329-5896-1

(Edition Sozialwirtschaft, Bd. 29)

Ausgewiesene Experten analysieren die aktuellen unternehmenspolitischen und strategischen Anforderungen, mit denen Sozial- und Gesundheitsunternehmen konfrontiert sind. Gestaltungsmöglichkeiten für den betrieblichen Einsatz der Steuerung in ökonomischen Fragen der Kosten-, Leistungs- und Qualitätssteuerung sowie des IT-Controllings und des RisikoHandlings werden erörtert.

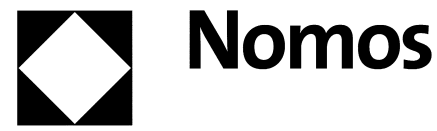

